\title{
ALGUNOS ASPECTOS DE LA DESCRIPCION Y ANALISIS DE UNA HACIENDA LOCAL
}

\author{
POR \\ MANUEL TORIBIO LEMES
}

\begin{abstract}
SUMARIO: I. INTRODUCCIÓN.-II. FunCIONES de las haciendas locales: A) Funciones generales. B) Funciones especificas. C) Criterios generales de financiación.III. Itinerario para la descripción y análisis de una hacienda local: A) Hacienda central de la entidad. B) Haciendas descentralizadas. C) Hacienda General.IV. ANÁlisis ECONÓmiCo-fINANCIERO: A) Ahorro bruto. B) Capacidad o necesidad de financiación. C) Saldo bruto a financiar. -V. INCIDENCIA EN LAS ECONOMIAS DOMÉSTICAS: A) Determinación de la renta per cápita local. B) Determinación de la presión, carga y esfuerzo fiscal locales.-VI. CONCLUSIón.
\end{abstract}

\section{INTRODUCCION}

La literatura sobre el sector de haciendas locales es abundante y valiosa en su aspecto jurídico, pero no se puede afirmar lo mismop del económico. Tampoco es ajeno a esta situación, que no es privativa del sector de haciendas locales, el criterio -comúnmente admitido- de que el estudio de las funciones de las Entidades Locales corresponde a las ciencias políticas y administrativas y no a la economía, que lo único que va a tener es un papel instrumental o de aportación de medios técnicos al servicio de fines políticos. No obstante, también se está haciendo notar en esta materia la especial atención a lo económico, que constituye una de las características definidoras de nuestra época, si bien los trabajos se refieren más a aspectos concretos o locales, objeto de tesis doctorales *, que a tratados o manuales.

Este trabajo pretende exponer algunas ideas generales sobre las haciendas locales y trazar el itinerario a seguir para la descripción y análisis de una hacienda local, tal como se ha realizado para el caso concreto de la del municipio de Sevilla en la década de los 70.

\section{FUNCIONES DE LAS HACIENDAS LOCALES}

Esta descripción y análisis ha de comenzar con la visión panorámica del cumplimiento por parte de la Entidad de las funciones generales y peculiares de la hacienda local a estudiar.

- El presente articulo es un análisis de la tesis doctoral leída por el autor en la Facultad de Ciencias Económicas y Empresariales de la Universidad de Sevilla, bajo el título de La Hacienda del municipio de Sevilla en la década de los 70. 


\section{A) Funciones generales}

Las primeras están representadas por sus deberes de colaboración con las políticas de la Hacienda Pública del Estado en las ramas de estabilización, redistribución, asignación de recursos y desarrollo.

Si bien en las tres primeras ramas las posibilidades de colaboración son de escasa entidad no ocurre lo mismo con la última, entendida como un proceso de crecimiento a consecuencia de la utilización del potencial de desarrollo existente en el área. Esta concepción está basada en la visión territorial del espacio económico.

\section{B) Funciones especificas}

La respuesta a la cuestión de ¿qué funciones específicas deben atribuirse a las Entidades Locales? ha sido dada por la doctrina partiendo de la clasificación de bienes con arreglo al principio de exclusión y aplicando criterios técnicos y económicos de localización. Frente a la tradicional clasificación de los bienes producidos por las Entidades Públicas en sociales, preferentes y oferta pública de bienes privados, la actual realidad social, obliga a las Administraciones públicas a introducir nuevos principios, lo que motiva la siguiente clasificación cuatripartita:

Bienes públicos. Aquellos en que no actúa el principio de exclusión, son indivisibles, no puede excluirse a quienes no paguen un precio, etc., es decir, aquellos que se corresponden con el concepto propio del término "público» como algo de disponibilidad general y gratuita. Constituyen ejemplos de los mismos en la esfera local, los servicios que implican ejercicio de autoridad, seguridad ciudadana, contra incendios, alumbrado público, parques públicos y otros de características similares.

Bienes sociales. Pueden ser producidos tanto por Entidades Públicas, como por Instituciones sociales o particulares. Satisfacen un tipo de necesidades que frecuentemente son atendidas también por fundaciones, asociaciones, organizaciones religiosas o particulares que actúan, predominantemente, con fines altruistas o de grupo. Su utilización no será, normalmente, general sino por un colectivo determinado. Mientras en algunos países la participación de las Entidades Públicas en la producción de estos bienes es predominante, en otros se limita prácticamente a garantizar el principio de igualdad de oportunidades. En la esfera municipal son ejemplo de ello los servicios relacionados con la cultura, asistencia social o beneficencia y análogos. 
Bienes preferentes. Satisfacen necesidades individuales, son divisibles, actúa el principio de exclusión, puede cobrarse un precio, generan economías externas, etc.; pero al mismo tiempo son de gran interés para la Comunidad. En este caso se encuentran los servicios de abastecimento de agua, transportes públicos, mercados, mataderos y otros.

Oferta pública de bienes privados. En determinadas circunstancias es necesario que las Entidades Públicas participen en el mercado de bienes privados, caracterizados por la plena efectividad del principio de exclusión, divisibilidad, pago de precio, etc. En nuestro entorno cultural la oferta de bienes privados por el sector público se realiza, normalmente, con sujeción al principio de subsidiariedad.

En cuanto a los criterios de localización se postula el de considerar la existencia de una estructura concéntrica de servicios públicos en áreas progresivamente crecientes, partiendo del muinicipio, según las premisas técnicas y económicas de cada uno de ellos (1). Tales premisas se concretan a través de los principios de eficacia y eficiencia.

A ellos ha venido a añadir la doctrina, y la realidad social más creciente, el criterio de mejor control por los ciudadanos a través de la votación, tanto en su funcionamiento como en su financiación.

\section{C) Criterios generales de financiación}

Está comúnmente admitido un sistema de financiación basado en impuestos para los bienes públicos; impuestos y tasas para los bienes sociales; precios públicos para los bienes preferentes $y$ precios de mercado para los bienes privados.

Las características de las necesidades públicas que aconsejan la prestación gratuita, da lugar a que el coste de producción de los servicios públicos que las satisfacen tenga que ser financiado por todos los vecinos, hagan o no demanda individual, mediante lo que Dalton llama "principio de la imposición general» (2).

Corolario de lo expuesto es la determinación de qué características han de tener los impuestos locales. Estas se resumen en que son idóneos los impuestos de incidencia localizada dentro de la jurisdicción y que permitan tener en cuenta tanto el principio de

(1) RAmón MARtín MAteo: La Comarcalización de los pequeños municipios, p. 27. Secretaría General Técnica del Ministerio de la Gobernación. Madrid. 1964.

(2) CAMILO LeBón FERnÁndeZ: La Hacienda del Municipio de Sevilla. Evolución y Crisis Actual. Publicaciones de la Universidad de Sevilla. Sevilla, 1976. 
beneficio como el de capacidad de pago (3). También es deseable que sean flexibles para adaptarse a la coyuntura económica.

A su vez esto conduce a dos cuestiones discutibles pero importantes: La libertad de las Entidades Locales para la fijación de los tipos impositivos y el denominado Impuesto Local sobre la Renta.

La libertad local en la fijación de los tipos impositivos de aquellos impuestos cuya recaudación está plenamente localizada, está admitida en países como Inglaterra, Francia, Alemania, Países Escandinavos o Suiza, para los impuestos inmobiliarios, e incluso, cuando existe, en el impuesto o recargo sobre la renta. La Conferencia de Poderes Locales y Regiones de Consejo de Europa viene recomendando el reconocimiento, con carácter general, de la libertad en la fijación de estos tipos. La doctrina está dividida en este punto.

En cuanto a los argumentos en favor de un impuesto o recargo local sobre la renta, se formulan desde distintas perspectivas: aplicación del principio de capacidad de pago, como cuestión de equidad fiscal; solución de la insuficiencia crónica de las Haciendas de las Ciudades; elevada elasticidad renta de los gastos locales; relación entre la demanda de los servicios que presta una Administración Local y el ritmo de crecimiento de la renta personal (4), y otros similares.

Otra alternativa, con la misma finalidad, es la postura de quienes, como el profesor García Añoveros (5), consideran más oportuno un impuesto local con la misma base que la del Impuesto sobre el Valor Añadido. Los argumentos en que se basa su preferencia, en relación al Impuesto Local sobre la Renta, como impuesto de ingreso flexible, son el de su más directa vinculación con el lugar de generación de la riqueza $y$, por tanto, con el de

(3) Históricamente, el principio del beneficio de la imposición deriva de la teoría contractual del Estado tal como se entendia por los teóricos políticos del siglo XVII, como LOCKE y HOBES. Más tarde se entrelazó con el principio de la máxima felicidad de los utilitaristas, como BENTHAM. Apareció pronto en la economia clásica, en la primera norma de la imposición de ADAM SMITH. que en una frase combina el punto de vista del beneficio con el de la capacidad de pago: "Los súbditos de todo Estado deberian contribuir al sostén del Gobierno lo más posible en proporción a sus respectivas capacidades; esto es, en propoción a los ingresos que respectivamente disfrutan bajo la protección del Estado.» Aqui los beneficios se contemplan en términos de la protección recibida y se encuentran, por tanto, relacionados con la renta, que a su vez es también una medida de la capacidad de pago.

Sobre las ventajas e inconvenientes de ambos principios, considerados separadamente, ver, entre otros, RichaRd A. MUSGRAVE y PEgGY B. MUSgRAVE: Hacienda Pública Teórica y Aplicada. Segunda edición, p. 750. Instituto de Estudios Fiscales. Madrid, 1983.

(4) SOlÉ VILlalongA. Citado por Eugenio Domingo Solans en el estudio introductorio de Comisión Layfield. La Reforma de las Haciendas Locales, pp. 27 y 28. Instituto de Estudios Fiscales. Madrid, 1982.

(5) Observación verbal como miembro del Tribunal que juzgó esta tesis doctoral. 
utilización de la infraestructura y servicios municipales ya que en el de la renta sería muy frecuente el caso de que el contribuyente cause los gastos públicos en un municipio distinto al de su residencia. Por otra parte este impuesto evita el peligro de distorsionar las políticas estabilizadoras centrales.

Dentro del capítulo de financiación mediante impuesto merece especial referencia la participación en los del Estado y en los de las Comunidades Autónomas, asunto tan importante que no faltan opiniones a favor de que los ingresos de naturaleza impositiva tengan, predominantemente, este origen por estimar que las Entidades Locales son más eficientes que otros niveles de Administraciones para realizar el gasto público pero menos para la gestión de ingresos.

\section{ITINERARIO PARA LA DESCRIPCION Y ANALISIS DE UNA HACIENDA LOCAL}

Se trata de lograr una representación completa y sistematizada de la hacienda municipal y de ponerla en relación con otras magnitudes que permitan valorar sus datos. Pero para llegar a este conocimiento y comparación del conjunto, se ha de pasar por una fase previa de descripción y análisis de cada una de sus partes, es decir, estudiar de manera particular la hacienda central de la Entidad y la de cada uno de sus servicios personalizados. La consolidación de los datos obtenidos constituye el instrumento adecuado para la fase de determinación de la estructura y análisis de la hacienda general.

\section{A) Hacienda Central de la Entidad}

En la actualidad esta hacienda se encuentra representada unitariamente por el presupuesto de la Entidad Local, pero en épocas anteriores estaba integrada por el presupuesto ordinario, los presupuestos extraordinarios de liquidación de deudas, el presupuesto especial de urbanismo, y los presupuestos extraordinarios propiamente dichos. Los dos primeros constituyen de hecho el presupuesto de funcionamiento, soporte de la hacienda ordinaria. El. especial de urbanismo es de carácter mixto. Los últimos deben ser tratados como hacienda extraordinarios, dada la utilización que se hacía de los mismos.

Para su descripción y análisis, se requiere una previa fase de ajuste de ingresos y gastos, a fin de eliminar aquellos que carecen de contenido económico real, y aplicar la técnica de la consolidación dada la interrelación de dichos presupuestos. 
Es necesario traducir los datos a pesetas constantes (6), para que realmente sean homogéneos y someterlos al correspondiente tratamiento estadístico.

\section{a) Hacienda de funcionamiento}

a') Presupuesto Ordinario más extraordinarios de liquidación de deudas

Se expone a continuación el itinerario seguido para la descripción y análisis del presupuesto de funcionamiento del Ayuntamiento de Sevilla en el período 1976-1979 que constituye una de las dos partes en que se dividió la década para su estudio. La elección de este período se debe a que al haberse producido durante estos años situaciones límite en las haciendas municipales, presenta unos perfiles tan acusados que puede mostrar mejor que ningún otro los principales aspectos de la descripción y análisis.

Los presupuestos ordinarios del Ayuntamiento de Sevilla en esta etapa nacían prácticamente "cautivos». Como ejemplo baste decir que cuando se forma el anteproyecto del de 1976, los gastos de personal representaban el 78 por 100 y los de la carga financiera el 20 por 100, con lo cual solamente quedaba disponible un 2 por 100 para el funcionamiento de los servicios externos; si a ello añadimos, con el carácter de anécdota, que en algunos servicios, como el de recogida domiciliaria de basuras y limpieza pública, se llegó a la "canibalización» de vehículos por falta de recursos económicos, para la adquisición de cubiertas, repuestos y accesorios, el cuadro no puede resultar más expresivo.

La primera observación, de carácter panorámico, es que en los cuatro años del período fueron adoptadas medidas presupuestarias excepcionales -en algunos casos heterodoxas- para mantener en funcionamiento los servicios (7).

(6) A tal efecto se estima conveniente utilizar como índice deflacionario el PIB al coste de los factores por considerar que es más adecuado para las magnitudes presupuestarias, ya que responde al coste de la corriente de bienes y servicios de nuestra economía, frente a otros indices, como el IPC, que son parciales. También presenta la ventaja de no incluir bienes y servicios producidos en otros paises eludiendo así la incidencia de los costes exteriores, a diferencia de lo que ocurre con el PNB.

(7) En 1976 fue autorizado un préstamo de 900 millones, a largo plazo, para financiar el presupuesto ordinario y un presupuesto extraordinario de liquidación de deudas de pesetas 266.386.885. En 1977 se recibe una ayuda excepcional del Fondo Nacional de Cooperación Municipal de 500 millones de pesetas y se reduce al 50 por 100 la aportación al presupuesto especial de urbanismo. En 1978 se autoriza un presupuesto extraordinario de liquidación de deudas de 1.054.647.918 pesetas, no se aprueba presupuesto especial de urbanismo y fue concedida mora en el pago de la carga financiera del BCLE. En 1979 se autoriza un presupuesto extraordinario de liquidación de deudas de 923.729 .595 pesetas; no se aprueba presupuesto especial de urbanismo y el Estado se hace cargo de la carga financiera en un 50 por 100. 
Como el presupuesto extraordinario de liquidación de deudas al 31 de diciembre de 1978 recogía las extrapresupuestarias generadas durante los ejercicios de 1977 y 1978, se ha considerado distribuido su importe entre ambos años para establecer la serie de los presupuestos de funcionamiento con lo cual se llega a la que aparece en el cuadro 1, una vez eliminados los gastos e ingresos que carecen de contenido económico real.

Sobre la base de los datos de dicho cuadro se ha realizado el ajuste estadístico de la serie de presupuestos de funcionamiento en pesetas constantes.

El ajuste lineal produce un coeficiente de determinación (R2) de 94,62 mientras que el ajuste parabólico presenta uno de 98,70 por lo cual se ha de tomar el ajuste parabólico como más expresivo de la realidad para establecer la proyección a años sucesivos.

CUADRO 1

\section{EVOLUCION DE LOS PRESUPUESTOS ECONOMICOS DE FUNCIONAMIENTO \\ (ORDINARIOS + EXTRAORDINARIOS DE LIQUIDACION DE DEUDAS) EN EL PERIODO 1976-1979, EN PESETAS CONSTANTES}

(Indice deflacionario PIB al coste de los factores. Base: 1970)

\begin{tabular}{c|c|c|c|c|c|c}
\hline \multicolumn{2}{|c|}{ Años } & $\begin{array}{c}\text { Presupuesto } \\
\text { de funcionamiento } \\
\text { en pesetas } \\
\text { corrientes }\end{array}$ & $\begin{array}{c}\text { Indice } \\
\text { deflacio- } \\
\text { nario }\end{array}$ & $\begin{array}{c}\text { Presupuesto } \\
\text { de funcionamiento } \\
\text { en pesetas } \\
\text { constantes }\end{array}$ & $\begin{array}{c}\text { Decremento } \\
\text { absoluto } \\
\text { anual }\end{array}$ & \multicolumn{2}{|c|}{$\begin{array}{c}\text { Decremento relativo. } \\
\text { porcentaje }\end{array}$} \\
\cline { 6 - 9 } & & & & & & \\
1976 & 3.145 .670 .573 & 2,0590 & 1.527 .766 .184 & - & - & - \\
1977 & 3.587 .553 .438 & 2,5311 & 1.417 .389 .055 & 110.377 .129 & 7.22 & 7.22 \\
1978 & 3.838 .731 .592 & 3,1013 & 1.237 .781 .443 & 179.607 .612 & 12,67 & 18.98 \\
1979 & 4.127 .191 .577 & 3,6162 & 1.141 .306 .226 & 96.475 .217 & 7.79 & 25.29 \\
\hline
\end{tabular}

FUENTE: Elaboración propia.

La verificación de la proyección establecida sobre la base de los datos de periodo 1971-1975 (8) presenta los resultados que refleja el cuadro 2 .

Este cuadro pone de manifiesto el efecto amortiguador de las medidas excepcionales, especialmente por los presupuestos extraordinarios de liquidación de dedudas, pero no pueden evitar la tendencia negativa, a la que hubo de hacer frente el Gobierno con el Real Decreto Ley $11 / 1979$, del 20 de julio, de medidas urgentes de financiación.

(8) Lógicamente, los resultados de la misma fueron los que aparecen en la columna de "serie proyectada", cuadro 3 . 


\section{VERIFICACION DE PROYECCIONES}

(Período base: 1971-1975)

\begin{tabular}{c|c|c|r|r}
\hline Años & $\begin{array}{c}\text { Serie proyectada } \\
(11)\end{array}$ & $\begin{array}{c}\text { Serie real } \\
(2)\end{array}$ & $\begin{array}{c}\text { Residuos }\left(e_{i}\right) \\
(1-2)\end{array}$ & $e_{i}{ }^{2}$ \\
\hline 1976 & $1.147,84$ & $1.527,8$ & $-379,96$ & $144.369,60$ \\
1977 & $1.297,76$ & $1.417,4$ & $-119,64$ & $14.313,73$ \\
1978 & $1.481,58$ & $1.237,8$ & 243,78 & $59.428,69$ \\
1979 & $1.699,30$ & $1.141,3$ & 558,00 & $311.364,00$ \\
\hline \multicolumn{1}{l}{$\Sigma$} \\
\hline
\end{tabular}

En millones de pesetas (Base: 1970).

b') Consolidación presupuesto de funcionamiento-presupuesto especial de urbanismo

El nutrirse el presupuesto especial de urbanismo del ordinario, en casi su totalidad -e incluso no haberse llegado a formar en 1978 y 1979-, da lugar a que la serie de datos de esta consolidación sea muy parecida a la de presupuestos de funcionamiento, pero se ha de efectuar para contrastar su evolución con la de otros índices expresivos de magnitudes económicas interrelacionadas. La serie en cuestión aparece en el cuadro 3 . Confirma las dificultades a que se ha aludido y el fenómeno de empobrecimiento progresivo del conjunto citado.

\section{c) Cobertura anual de inflación}

Es indudable que la comparación de incrementos del presupuesto consolidado, en pesetas corrientes, con los del IPC de cada ejercicio, refleja su grado de capacidad para dar cobertura a la inflación. Los datos representativos de ambas series, que se exponen a continuación, indican que dicha cobertura solamente se logró en 1976, a pesar de las aludidas medidas excepcionales.

\begin{tabular}{c|c|c|c}
\hline Año & $\begin{array}{c}\text { Porcentaje } \\
\Delta \text { anual } \\
\text { presupuestario }\end{array}$ & IPC & $\begin{array}{c}\text { Cobertura anual } \\
\text { de la inflación }\end{array}$ \\
\hline 1976 & 74,85 & 17,60 & 57,25 \\
1977 & 14,05 & 24,50 & $-10,45$ \\
1978 & 6,97 & 19,80 & $-12,83$ \\
1979 & 7,52 & 15,70 & $-8,18$ \\
\hline
\end{tabular}


CUADRO 3

EVOLUCION DE LA CONSOLIDACION ECONOMICA DE LAS PREVISIONES DE INGRESOS EN EL PERIODO $1976-1979$

\begin{tabular}{|c|c|c|c|c|c|c|c|}
\hline \multirow{2}{*}{$\begin{array}{c}\begin{array}{c}\text { Con- } \\
\text { ceptos }\end{array} \\
\text { Años }\end{array}$} & \multicolumn{3}{|c|}{ Pesetas corrientes } & \multicolumn{4}{|c|}{ Pesetas constantes. Indice PIB al coste de los factores. Base: 1970} \\
\hline & Importe & Incremento anual & Porcentaje & $\begin{array}{l}\text { Indice de- } \\
\text { flacionario }\end{array}$ & Importe & Incremento anual & Porcentaje \\
\hline $\begin{array}{l}1976 \\
1977 \\
1978 \\
1979 \\
\end{array}$ & $\begin{array}{l}3.146 .420 .473 \\
3.588 .318 .338 \\
3.838 .731 .592 \\
4.127 .191 .577\end{array}$ & $\begin{array}{c}1.346 .955 .663 \\
441.898 .394 \\
250.413 .254 \\
288.459 .985\end{array}$ & $\begin{array}{r}74,85 \\
14,05 \\
6,97 \\
7,52\end{array}$ & $\begin{array}{l}2,0590 \\
2,5311 \\
3,1013 \\
3,6162\end{array}$ & $\begin{array}{l}1.528 .130 .389 \\
1.417 .691 .255 \\
1.237 .781 .443 \\
1.141 .306 .227\end{array}$ & $\begin{array}{r}501.097 .223 \\
-110.439 .134 \\
-179.909 .812 \\
-\quad 96.475 .216\end{array}$ & $\begin{array}{r}48,7908 \\
-\quad 7,2271 \\
-12,6903 \\
-\quad 7.7942\end{array}$ \\
\hline \multicolumn{2}{|c|}{ Incremento en el periodo: } & 2.327 .726 .767 & 129,35 & & & & \\
\hline
\end{tabular}

- Se parte de un importe de 1.799.464.810 pesetas corrientes en 1975, equivalentes a 1.027.033.166 pesetas constantes. 
d') Participación en el PIB

La serie de incrementos anuales, en pesetas constantes, comparada con los del PIB, al coste de los factores, en cada uno de los ejercicios, explica la precaria situación económica, agravada por partir ya de unos incrementos presupuestarios negativos.

\begin{tabular}{|c|c|c|c|}
\hline Año & $\begin{array}{c}\text { Porcentaje } \\
\Delta \text { anual } \\
\text { presupuesto }\end{array}$ & $\begin{array}{l}\Delta \text { anual del PIB al } \\
\text { coste de los fact. }\end{array}$ & $\begin{array}{l}\text { Cobertura } \\
\text { de la financiación }\end{array}$ \\
\hline $\begin{array}{l}1976 \\
1977 \\
1978 \\
1979\end{array}$ & $\begin{array}{r}48,79 \\
-\quad 7.22 \\
-12.70 \\
-\quad 7.79\end{array}$ & $\begin{array}{l}3,00 \\
2,57 \\
2,91 \\
1,05\end{array}$ & $\begin{array}{r}45,79 \\
-\quad 9,79 \\
-\quad 15,61 \\
-\quad 8,84\end{array}$ \\
\hline
\end{tabular}

En ambas comparaciones se produce además otro factor de distorsión cual es la dispersión de los saldos resultantes, a pesar de que los presupuestos extraordinarios de liquidación de deudas tenían como objetivo corregir los desequilibrios.

Pero, posiblemente, la forma más gráfica de expresar el proceso de empobrecimiento progresivo en que se encontraba la hacienda municipal, venga dada por los resultados de la proyección de la serie de presupuestos de funcionamiento para el período 1980-1984, en pesetas constantes, que aparece en la columna 6 del cuadro 4 , y que se hubiera producido de continuar los elevados índices anuales de inflación, y de no haberse mejorado el cuadro de ingresos, cosa que se inició con el citado Real Decreto Ley 11/1979, del 20 de julio, de medidas urgentes de financiación.

CUADRO 4

PROYECCIONES DE LA SERIE SEGUN AJUSTE PARABOLICO SERIE: 1976-1979

(Importe en millones de pesetas. Base: 1970)

\begin{tabular}{c|c|c|c|c|c}
\hline 1 & 2 & 3 & 4 & 5 & 6 \\
\hline$t$ & $t_{i}^{\prime}$ & $t_{i}^{2}$ & $133,92 \times t_{i}$ & $3,44 \times t_{i}^{2}$ & $P_{F i}=1.326,77-(4)+(5)$ \\
\hline 1980 & 2,5 & 6,25 & 334,80 & 21,50 & $1.013,47$ \\
1981 & 3,5 & 12,25 & 468,72 & 42,14 & 900,19 \\
1982 & 4,5 & 20,25 & 602,64 & 69,66 & 793,79 \\
1983 & 5,5 & 30,25 & 736,56 & 104,06 & 694,27 \\
\hline
\end{tabular}

$t_{i}^{\prime}(t-1.977,5)$. 


\section{b) Hacienda extraordinaria}

Durante el período 1976-1979 solamente tuvo efectividad un presupuesto extraordinario de 115.071 .915 pesetas corrientes, equivalente en pesetas constantes de 1970, a 35.711 .742 pesetas, lo que indica la práctica paralización de inversiones en esta etapa debido a la situación de mera supervivencia en que se encontraba la hacienda municipal.

\section{B) Haciendas descentralizadas}

a) Consideraciones generales: centralización versus descentralización

La existencia de entes públicos y privados, en los que se descentraliza la prestación de determinados servicios, o la realización de actividades de la competencia Local, dotados de personalidad jurídica propia, da lugar a que cada uno de ellos cuente con recursos económicos propios.

Es evidente que las haciendas de estos entes -que se podrian denominar hacienda descentralizadas - forman parte de la actividad económico-financiera que genera el Ayuntamiento.

La cuestión que plantea al economista la existencia de estos entes, desde la perspectiva que le es propia, es la de su justificación o condicionamientos. La respuesta viene dada, principalmente, por las técnicas de organización y los costes, es decir, por aspectos de eficacia y eficiencia. En definitiva se trata de decidir entre centralización o descentralización, lo que conecta lo económico con criterios organizativos tales como el principio de coordinación y el poder de decisión.

La centralización presenta inconvenientes en las grandes estructuras al llegar los problemas a los órganos de la alta dirección que se ven obligados a decidir sobre asuntos que en muchos casos ni conocen ni comprenden por lo que resuelven de forma lenta $y, a$ veces, desacertada.

La respuesta común a este problema es invertir el proceso, es decir, descentralizar para permitir que los problemas encuentren solución a niveles inferiores dentro de la jerarquía de la organización (9) de esta manera las decisiones son más rápidas y se toman en el lugar en que se encuentra el problema por lo que se apoyan en una información más directa.

Pero la descentralización no es una panacea. Ni en todos los casos funciona bien ni siempre implica ventajas.

(9) DAVID R. HAMPTON: Administración contemporánea, p. 312. Libros MacGraw Hill de México, S. A. C. V. Maucalpan de Juárez, 1987. 
Entre los beneficios que se pueden obtener de la descentralización cabe mencionar los siguientes:

- Concreta la responsabilidad del funcionamiento.

- Fortalece la autoridad del responsable.

- Perfecciona la toma de decisiones por el conocimiento más directo de la realidad.

- Permite una respuesta más rápida a los problemas.

- Favorece la especialización.

- Estimula la innovación.

- Aumenta el rendimiento, etc.

En cambio presenta desventajas como las siguientes:

- Produce la inhibición frente a los problemas generales de la Entidad, que llega a agravar, o a perturbar, con tal de alcanzar el éxito de su unidad.

- Se pierde el sentido de cooperación con las demás unidades.

- Cuando el problema es muy grave, o complejo, las unidades descentralizas se las arreglan para dirigirlo a los Organos centrales.

- Generalmente el aumento de eficacia se logra a costa de minorar la eficiencia.

- Incrementa los costes del servicio tanto por la necesidad de contar con administración propia como por los aumentos habituales de plantilla y de salarios.

- Dificulta la coordinación, con la consiguiente dispersión de recursos y pérdidas de fuerza frente al exterior.

Ante estos catálogos de ventajas e inconvenientes cabe formularse la siguiente pregunta: ¿cuándo debe descentralizarse una superestructura? La respuesta normal será la de que sólo cuando no sea posible mantenerla centralizada. A lo que habría que añadir, en el caso de las entidades públicas, que cuando el organismo autónomo o-empresa generen sus propios recursos, particularmente en el caso de las últimas.

La primera cuestión que se plantea, pues, al describir o analizar las haciendas descentralizadas, es pronunciarse sobre las razones que justifican su existencia.

En el Ayuntamiento de Sevilla existían en la década de los setenta las siguientes fundaciones públicas del servicio (hoy Organismos Autónomos) y empresas municipales:

- Patronato Municipal de la Vivienda (PMV), para la gestión de las competencias en materia de vivienda.

- Empresa Municipal de Abastecimiento de Aguas y Saneamiento de Sevilla, S. A. (EMASESA), para la gestión de las 
competencias en materia de abastecimiento de aguas y saneamiento.

- Transportes Urbanos de Sevilla, SAM (TUSSAM), para la gestión de las competencias que indica su denominación.

- Mercasevilla, S. A., empresa mixta, concesionaria de las competencias municipales en materia de abastos y matadero municipal.

\section{b) Evolución de las haciendas descentralizadas}

La descripción y análisis de las haciendas descentralizadas se realiza tanto desde una vertiente puramente empresarial, que permite enjuiciar la gestión (10), como de la presupuestaria, para integrar sus datos en la hacienda general por vía de consolidación.

En el primero de dichos sentidos se estudia la evolución de las magnitudes más características: tales como financiación básica, aplicaciones e inversiones, explotación y resultados, como mínimo.

Desde el punto de vista presupuestario el método consiste en equiparar los conceptos de gastos e ingresos empresariales a sus similares presupuestarios, con el fin de incluirlos en la hacienda general, tanto para conocer el volumen de aquélla como para poner de relieve el peso de cada hacienda descentralizada dentro del conjunto. Estos datos y operaciones se exponen en el epígrafe de la hacienda general.

\section{C) Hacienda general}

\section{a) Consideraciones preliminares}

La consolidación de la hacienda central con las descentralizadas constituye la fase de concreción y sistematización de la hacienda general. Es instrumento esencial para el análisis homogéneo de las distintas unidades que la componen, del peso de cada una de ellas y de las características económicas del conjunto, es decir, de la estructura real que tendría la hacienda de la Entidad de no existir las descentralizadas.

Pero existen también otras causas que hacen necesaria la integración contable de las haciendas de una entidad territorial.

La dinámica de penetración del sector público en las actividades económicas y sociales ha ampliado de tal modo su campo de actuación que, incluso en las de contacto más inmediato con la realidad física -provincia y municipio-, las decisiones se han de

(10) En el caso de las empresas municipales entendemos que la Intervención Municipal ha de realizar la correspondiente auditoria anual a fin de comprobar que los estados financieros reflejan la imagen real de la empresa. 
tomar a la vista de una información compleja y no de forma intuitiva. Por tanto, la necesidad de una información sistematizada y completa - veraz- es evidente, en unas entidades que han pasado «de realizar un papel puramente de cobertura administrativa, a ser protagonista de la economía, en sentido amplio» (11). Una información parcial o sesgada difícilmente permite prever las consecuencias de las decisiones.

\section{b) Consolidación: hacienda general}

Aplicando los criterios citados se ha obtenido el cuadro número 5 que refleja los totales anuales de derechos liquidados $y$ obligaciones reconocidas de la hacienda general. En él se puede observar que el comportamiento de la hacienda general es distinto que el de la central, descrito en páginas anteriores. Los saldos de derechos liquidados con relación a obligaciones reconocidas, en operaciones corrientes, son favorables a los primeros lo que implica financiación de operaciones de capital con ahorro. Esto indica que se encuentran descentralizados servicios económicamente productivos.

Los saldos totales negativos obedecen a que se ha operado solamente con las operaciones propias del ejercicio corriente. La concentración de los mismos en los últimos ejercicios de la década indican un empobrecimiento real de la hacienda municipal, ya que no puede ser neutralizado ni con las aportaciones de las descentralizadas de signo positivo. A ello se suma la circunstancia de que los presupuestos extraordinarios de liquidación de deudas no cubrieron en su integridad los déficit de alguna de las haciendas descentralizadas.

El proceso de empobrecimiento es una realidad evidenciada por el cuadro 6 , de evolución en pesetas constantes, que presenta en 1979 datos inferiores a los de 1971.

\section{c) Aportación de cada hacienda a la general}

A fin de apreciar la aportación de cada hacienda a la general se ha confeccionado el cuadro 7 que presenta los totales anuales de ingresos y gastos, en forma porcentual y comparativa, de cada uno de ambos conceptos dentro de cada ejercicio.

El examen de dicho cuadro pone de manifiesto la importancia de las haciendas descentralizadas como integrantes de la hacienda

(11) José MARÍA fernández PIRLA: En Estudio sobre Economia de las Corporaciones Locales II, p. 298. Centro de Estudios Municipales y Cooperación Interprovincial. Granada, 1984. 
RESULTADOS DE LAS OPERACIONES REALIZADAS POR LA HACIENDA GENERAL

(En millones de pesetas)

\begin{tabular}{|c|c|c|c|c|c|c|c|c|c|}
\hline \multicolumn{10}{|c|}{ CONCEPTOS } \\
\hline \multirow{2}{*}{ Años } & \multicolumn{3}{|c|}{ Operaciones corrientes } & \multicolumn{3}{|c|}{ Operaciones de capital } & \multicolumn{3}{|c|}{ Totales anuales } \\
\hline & Derechos & Obligaciones & Saldo & Derechos & Obligaciones & Saldo & Derechos & Obligaciones & Saldo \\
\hline \multirow[t]{2}{*}{$\begin{array}{l}1971 \\
1972 \\
1973 \\
1974 \\
1975 \\
1976 \\
1977 \\
1978 \\
1979\end{array}$} & \multirow[t]{2}{*}{$\begin{array}{l}1.566,02 \\
1.756,48 \\
2.022,82 \\
2.810,34 \\
2.862,35 \\
4.660,79 \\
4.654,42 \\
6.143,82 \\
6.801,77\end{array}$} & \multirow[t]{2}{*}{$\begin{array}{l}1.381,12 \\
1.490,52 \\
1.721,62 \\
2.246,55 \\
2.669,23 \\
3.948,85 \\
4.379,36 \\
5.980,31 \\
6.532,30\end{array}$} & \multirow[t]{2}{*}{$\begin{array}{l}184,90 \\
265,96 \\
301,20 \\
563,79 \\
193,12 \\
711,94 \\
275,06 \\
163,51 \\
269,47\end{array}$} & \multirow[t]{2}{*}{$\begin{array}{r}849,19 \\
753,87 \\
275,20 \\
1.595,43 \\
126,70 \\
1.501,42 \\
306,88 \\
668,98 \\
1.393,66\end{array}$} & \multirow[t]{2}{*}{$\begin{array}{r}1.172,97 \\
1.014,10 \\
539,43 \\
2.049,17 \\
474,63 \\
1.387,10 \\
830,80 \\
1.429,51 \\
1.709,40\end{array}$} & \multirow[t]{2}{*}{$\begin{array}{r}-278,78 \\
-260,23 \\
-264,23 \\
-453.74 \\
-347.93 \\
114.32 \\
-523.92 \\
-760,53 \\
-315.74\end{array}$} & $\begin{array}{l}2.460,21 \\
2.510,35 \\
2.298,02 \\
4.405,77 \\
2.989,05 \\
6.162,21 \\
4.961,30 \\
6.812,80 \\
8.195,43\end{array}$ & $\begin{array}{l}2.554,09 \\
2.504,62 \\
2.261,05 \\
4.295,72 \\
3.143,86 \\
5.335,95 \\
5.210,16 \\
7.409,82 \\
8.241,70\end{array}$ & $\begin{array}{r}93,88 \\
5,73 \\
36,97 \\
100,05 \\
-154,81 \\
826,26 \\
-248,86 \\
-597.02 \\
-\quad 46,27\end{array}$ \\
\hline & & & & & & & $40.795,14$ & $40.956,97$ & $-161,83$ \\
\hline
\end{tabular}


CUADRO 6

EVOLUCION DE LA HACIENDA GENERAL EN PESETAS CONSTANTES

\begin{tabular}{r|c|r|r|r|r}
\hline \multirow{2}{*}{ Años } & \multicolumn{2}{|c|}{ Obligaciones reconocidas } & \multicolumn{2}{c|}{ Derechos reconocidos } & \multicolumn{1}{c}{$\begin{array}{c}\text { Indice } \\
\text { deflacionario }\end{array}$} \\
\cline { 2 - 5 } & (A) & Porcentaje & (B) & Porcentaje & \multicolumn{1}{c}{ ( } \\
\hline 1971 & $2.360,53$ & - & $2.273,76$ & - & 1,0820 \\
1972 & $2.147,12$ & $-9,04$ & $2.152,04$ & $-5,35$ & 1,1665 \\
1973 & $1.751,26$ & $-18,43$ & $1.779,89$ & $-17,29$ & 1,2911 \\
1974 & $2.863,05$ & 63,48 & $2.936,40$ & 64,97 & 1,5004 \\
1975 & $1.794,34$ & $-37,32$ & $1.705,98$ & $-41,90$ & 1,7521 \\
1976 & $2.591,53$ & 44,42 & $2.992,82$ & 75,43 & 2,0590 \\
1977 & $2.058,46$ & $-20,56$ & $1.960,14$ & $-34,50$ & 2,5311 \\
1978 & $2.389,26$ & 16,07 & $2.196,76$ & 12,07 & 3,1013 \\
1979 & $2.279,11$ & $-4,61$ & $2.266,31$ & 3,16 & 3,6162 \\
\cline { 2 - 5 } & & & & & \\
Total & & & & & \\
década & $20.234,66$ & $-3,44$ & $20.264,00$ & $-0,32$ & \\
\hline
\end{tabular}

FUENTE: Elaboración propia.

general, y el papel primordial, dentro de ellas, de TUSSAM y EMASESA.

Como se puede observar, en algunos ejercicios, los porcentajes de derechos liquidados y obligaciones reconocidas por las haciendas descentralizadas se mueven en una banda próxima al 50 por 100 de las operaciones de la hacienda general, lo que implica un reparto prácticamente a partes iguales entre hacienda central y haciendas descentralizadas.

Una reflexión inmediata es la necesidad de que las haciendas descentralizadas tengan la adecuada coordinación y control de los órganos generales de la entidad. No es fácil explicar que sobre un 50 por 100 de la hacienda general se ejerza un control y fiscalización minucioso, que de no ejercerse con prudencia encorseta las actividades, mientras que el otro 50 por 100 se rige, prácticamente, de forma feudalizada.

\section{d) Ingresos y gastos}

El análisis de la estructura de ingresos y gastos de la hacienda general determina las características de la misma.

En el caso concreto de la hacienda municipal de Sevilla el predominio de los ingresos derivados de la prestación de servicios (tasas y precios públicos), la clasifican como hacienda de servicios, $y$ el de los derivados de préstamos, para financiar las inversiones, como hacienda dependiente del exterior. 
EVOLUCION PORCENTUAL DE LOS DERECHOS LIQUIDADOS Y LAS OBLIGACIONES RECONOCIDAS EN LAS HACIENDAS QUE INTEGRAN LA GENERAL

\begin{tabular}{|c|c|c|c|c|c|c|}
\hline \multirow{2}{*}{ Año } & \multirow{2}{*}{$\begin{array}{l}\text { Hacienda } \\
\text { central (a) }\end{array}$} & \multicolumn{4}{|c|}{ Haciendas descentralizadas } & \multirow{2}{*}{ Suma (b) } \\
\hline & & PMV & EMASESA & TUSSAM & Mercasevilla & \\
\hline 1971 & $\begin{array}{r}48,99 \\
52,16\end{array}$ & $\begin{array}{r}15,03 \\
13,71\end{array}$ & 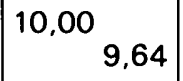 & \begin{tabular}{|l|}
14,95 \\
14,41
\end{tabular} & \begin{tabular}{|l|}
11,03 \\
10,08
\end{tabular} & $\begin{array}{r}51,01 \\
47,84\end{array}$ \\
\hline 1972 & $\begin{array}{r}70,15 \\
69,29\end{array}$ & $\begin{array}{r}0,21 \\
0,16\end{array}$ & \begin{tabular}{|l|}
10.71 \\
\\
10,74
\end{tabular} & \begin{tabular}{|l|}
15,01 \\
14,67
\end{tabular} & $\begin{array}{r}3,92 \\
5,14\end{array}$ & $\begin{array}{r}29,85 \\
30,71\end{array}$ \\
\hline 1973 & $\begin{array}{r}62,85 \\
62,16\end{array}$ & $\begin{array}{r}0,19 \\
0,24\end{array}$ & \begin{tabular}{|l|}
13,26 \\
13,55
\end{tabular} & \begin{tabular}{|r|}
18,93 \\
19,65
\end{tabular} & $\begin{array}{r}4,77 \\
4,40\end{array}$ & $\begin{array}{r}37,15 \\
37,84\end{array}$ \\
\hline 1974 & \begin{tabular}{|}
74,66 \\
74,84
\end{tabular} & $\begin{array}{r}0,37 \\
0,17\end{array}$ & $\begin{array}{rr}9,01 & \\
& 9,50\end{array}$ & \begin{tabular}{|l|}
12,37 \\
12,57
\end{tabular} & $\begin{array}{r}3,59 \\
2,92\end{array}$ & $\begin{array}{r}25,34 \\
25,16\end{array}$ \\
\hline 1975 & $\begin{array}{r}62,06 \\
60,49\end{array}$ & $\begin{array}{r}0,26 \\
0,40\end{array}$ & \begin{tabular}{|c|}
14,09 \\
15,99
\end{tabular} & \begin{tabular}{|l|}
16,85 \\
16,32
\end{tabular} & $\begin{array}{r}6,74 \\
6,80\end{array}$ & $\begin{array}{r}37.94 \\
39,51\end{array}$ \\
\hline 1976 & \begin{tabular}{|}
53,06 \\
57,09
\end{tabular} & $\begin{array}{r}10,12 \\
11,31\end{array}$ & $\left.\right|_{11,13} ^{19,50}$ & \begin{tabular}{|l|}
13,41 \\
\\
\\
15,31
\end{tabular} & $\begin{array}{rr}3,91 & \\
& 5,16\end{array}$ & $\begin{array}{r}46,94 \\
42,91\end{array}$ \\
\hline 1977 & \begin{tabular}{|c|}
61,81 \\
58,61
\end{tabular} & ${ }_{4,70}^{5,25}$ & \begin{tabular}{|c|}
11,50 \\
15,37
\end{tabular} & \begin{tabular}{|c|}
16,35 \\
16,22
\end{tabular} & $\begin{array}{r}5,09 \\
5,10\end{array}$ & $\begin{array}{r}38,19 \\
41,39\end{array}$ \\
\hline 1978 & \begin{tabular}{|}
63,45 \\
59,18
\end{tabular} & $\begin{array}{r}0,86 \\
0,83\end{array}$ & \begin{tabular}{|}
17,05 \\
20,26
\end{tabular} & \begin{tabular}{|l|}
13,89 \\
15,99
\end{tabular} & $\begin{array}{r}4,75 \\
3,74\end{array}$ & $\begin{array}{r}36,55 \\
40,82\end{array}$ \\
\hline 1979 & \begin{tabular}{|}
56,45 \\
54,58
\end{tabular} & $\begin{array}{r}5,85 \\
5,80\end{array}$ & \begin{tabular}{|}
15,85 \\
20,30
\end{tabular} & $\begin{array}{c}17,78 \\
15,49\end{array}$ & $\begin{array}{r}4,07 \\
3,83\end{array}$ & $\begin{array}{r}43,55 \\
45,42\end{array}$ \\
\hline
\end{tabular}

Nota: $a+b=100$ por 100 .

DERECHOS

LIQUIDADOS

OBLIGACIONES RECONOCIDAS 
La vertiente de gastos muestra el predominio de los de personal. Merecen también especial atención los de carga financiera, por su naturaleza.

La integración de los datos del número de personas que trabajan para la entidad, sus organismos autónomos y empresas municipales es básica para enjuiciar este gasto.

Al relacionar el total de personas empleadas con el gasto total y con el número de habitantes se obtienen unos «ratios» que permiten establecer comparaciones nacionales similares. No ocurre lo mismo con las extranjeras, ya que para ello se requeririan "ratios» sintéticos, dadas las diferencias de competencias, renta per cápita $y$ otros datos sin los cuales la comparación carece de rigor.

La evolución del endeudamiento y de la carga financiera se ha de analizar tanto en su evolución como en relación con la del presupuesto, población y otros aspectos significativos.

\section{ANALISIS ECONOMICO-FINANCIERO}

El análisis económico-financiero se realiza sobre valores consolidados de ingresos y gastos y de ellos se deducen varias magnitudes que describen la situación general de la hacienda local.

El profesor Fuentes Quintana, a efectos del estudio de los ingresos desde el punto de vista económico, en el caso del Estado, los distribuyen según «afecten a la cuenta de renta, a la cuenta de capital o a la cuenta financiera del país» (12).

Trasladamos dicho análisis a la hacienda municipal con arreglo a sus peculiaridades.

\section{A) Ahorro bruto}

Corresponde a la diferencia entre ingresos corrientes (a excepción de las contribuciones especiales) y gastos corrientes; representa pues la variación que ha sufrido el patrimonio de dicho agente en el período considerado, excepción hecha de las ganancias $y$ pérdidas de capital que no se recogen en estas cuentas. Supone en realidad el volumen de recursos destinados a la financiación de operaciones de capital (ahorro positivo) y la parte de gastos corrientes que debe ser financiada con ingresos $u$ operaciones de capital (desahorro); es una magnitud, pues, importante, ya que establece el grado de maniobrabilidad de la hacienda sobre sus valores de conjunto.

(12) Ver EnRIQue fuentes Quintana: Hacienda Pública. Principios y Estructura de la Imposición, p. 19. Rufino Blanco. Madrid, 1986. 


\section{B) Capacidad o necesidad de financiación}

En el primer caso supone el volumen de recursos en que el ahorro y los ingresos de capital exceden de los gastos de capital. En el caso contrario supone la medida en que será necesario acudir al mercado financiero para la obtención de recursos.

Entre los ingresos de capital solamente consideramos las contribuciones especiales, por su carácter finalista, y la enajenación de inversiones reales, eludiendo en este caso la imposición sobre el capital, ya que al considerar que ésta es la que grava la titularidad en su valor, no encontramos ninguna figura digna de tenerse en cuenta a estos efectos. Debemos indicar que no consideramos incluible en tal concepto el arbitrio o impuesto sobre el incremento del valor de los terrenos, ya que grava la transmisión, o la posibilidad de transmisión en la modalidad de tasa de equivalencia, lo que lo asimila más al de transmisiones patrimoniales de la hacienda estatal o autonómica, aun cuando se diferencien en que este último no tiene en cuenta el tiempo transcurrido. Desde el punto de vista económico este arbitrio lo que grava, a nuestro juicio, es una renta acumulada a lo largo del tiempo en forma de incremento de valor. Otro ingreso al que se debe hacer referencia en este punto es al impuesto sobre solares, pero del que prescindimos a estos efectos tanto por su escasa significación como por los fines realmente perseguidos.

En una hacienda como la municipal, en la que los ingresos de capital son mínimos, el ahorro debe jugar un papel importante ya que su inexistencia supondrá, en la mayoría de los casos, un cargo importante en la cuenta financiera (necesidad de financiación). De ahí que la capacidad de ahorro sea pieza clave para la autofinanciación de la hacienda municipal.

\section{C) Saldo bruto a financiar}

Representa los valores reales de la necesidad de financiación tanto por operaciones de capital como para complementar las restantes obligaciones financieras. Determina, pues, el nivel de endeudamiento a que se ve forzada la hacienda municipal.

En última instancia, supone el grado de dependencia de la hacienda estudiada, ya que hace necesario acudir a sectores externos para la financiación del mencionado saldo. De ahí que un valor negativo supusiese una completa autofinanciación de las operaciones presupuestarias, y la posibilidad de reciclar el excedente a otras unidades, y un valor positivo determine el nivel de recursos externos que es necesario obtener. 


\section{INCIDENCIA EN LAS ECONOMIAS DOMESTICAS}

En un sistema de hacienda multijurisdiccional como el que corresponde a los estados federales y autonomistas, donde cuentan con potestades tributarias - de distinta naturaleza- el Estado, las Comunidades Autónomas, las Entidades Locales y las Institucionales, parece necesaria la fijación del límite de carga fiscal de los dintintos niveles de haciendas (13). La necesidad de prestar atención a este aspecto se formula por la doctrina tanto desde la perspectiva de la distribución adecuada de la capacidad tributaria de los contribuyentes entre los distintos niveles de haciendas, federalismo fiscal, como dato a tener en cuenta a efectos de equilibrio presupuestario. En este último sentido, se sostiene el criterio de que «debe valorarse la presión fiscal del municipio y su capacidad para financiar mediante impuestos el porcentaje del precio de referencia (coste medio), no cubierto mediante el precio, pues el equilibrio presupuestario como objetivo de gestión en la prestación del servicio es la única alternativa para producir en condiciones de eficiencia y rentabilidad social» (14).

La determinación de dicha carga fiscal requiere el previo conocimiento de la renta local.

\section{A) Sistemas de determinación}

No parece viable la determinación directa de la renta personal en las áreas de las haciendas subnacionales desde ninguno de los tres clásicos enfoques de renta producida, percibida o gastada, por tratarse de economías abiertas «definidas por límites muchas veces puramente administrativos y sobre los que no existe prácticamente control. Estas características suelen ser tanto más acusadas cuanto menor es el área. Así, las ciudades son, en realidad, como unidades totalmente abiertas, situadas en verdaderas encrucijadas de factores tales como el trabajo y el capital. Ello significa, en definitiva, que el valor añadido o renta generada en una zona urbana puede diferir sustancialmente, de la renta percibida por sus residentes habituales y de la gastada en dicha zona» (15), lo que implica también la

(13) A tal efecto, sería oportuno que uno de los documentos obligatorios que deben acompañar a los presupuestos anuales de las Comunidades Autónomas y de las Entidades Locales fuese el estudio de la estimación de la renta personal de su demarcación territorial y la determinación de la carga tributaria en función de la misma.

(14) JOSÉ VALLÉS FERRER: Los servicios públicos locales: costes, financiación y tamaño de población, p. 193. Obra Cultural Caja de Ahorros Provincial San Fernando. Sevilla, 1986.

(15) FERNANDO ARAMBURU CAMPOY: Metodología para la estimación de la renta personal disponible a nivel municipal, comarcal o metropolitano, p. 23. Instituto de Estudios de Administración Local. Madrid, 1975. 
necesidad de delimitar el concepto de renta, de entre los tres citados, con el que se va a trabajar.

El método a adoptar debe proponerse, como objetivo, estimar la cuantía del concepto de renta que mejor se adecue al fin perseguido, a partir de unos datos básicos de referencia, mediante la selección de los indicadores más representativos y el establecimiento de los coeficientes de correlación en función de los mismos.

En nuestro caso, al tratarse de estimar la renta por razones fiscales, es oportuno utilizar la modalidad de ingreso percibido. «En general puede decirse que la renta de un núcleo urbano, medida a través de los ingresos percibidos por su población residente, es la más comúnmente aceptada como expresión de la riqueza de la colectividad, por el enfoque concreto que vemos adquiere la actividad de los grandes núcleos urbanos en los momentos actuales» (16).

Los datos de referencia, básicos para proceder a estimar la renta percibida por los residentes de una ciudad en nuestro país, pueden ser los proporcionados a nivel provincial por el Banco de Bilbao, que gozan de general aceptación. El mejor camino es proceder a través de un método estimativo de ingresos de tipo indirecto, basado en el sistema de indicadores directos de gasto. En este sentido consideramos como adecuados los indicadores de motorización (automóviles de turismo) y los de consumo de electricidad para usos domésticos (tarifas A-1 y A-2), en kWh, por su reconocida relación con los niveles de renta personales.

En el caso estudiado se realizó un primer ajuste de valores provinciales y municipales, de renta interior y renta interior per cápita, utilizando la población de derecho al 31 de diciembre del año considerado.

Un segundo ajuste consistió en determinar los valores de renta personal y disponible y per cápita, siendo ésta, pues, el último tramo de renta que se destina al consumo o al ahorro. Estos datos bianuales se obtuvieron a partir de los de la publicación del Servicio de Estudios del Banco de Bilbao.

\section{B) Presión, carga y esfuerzo fiscal local del municipio de Sevilla}

\section{a) Presión fiscal}

La presión fiscal local y su evolución en el período 1971-1979 será el cociente entre ingresos coactivos municipales y la renta

(16) Fernando aramburu Campoy: Ob. cit., p. 30. 
interior municipal. Presenta varias modalidades, entre las que se pueden considerar más significativas las siguientes:

Presión fiscal tributaria. Es el índice resultante de dividir los ingresos tributarios por la renta interior del municipio.

Presión fiscal financiera. Este índice expresa la relación existente entre los ingresos totales del municipio y la renta interior del municipio. Representa, por tanto, el volumen de fondos que se detraen del sistema financiero.

La valoración de ingresos municipales se realizará sobre valores liquidados en lugar de recaudados por la falta de sincronización entre devengos y cobros-pagos.

La utilización en el denominador de la Renta Interior Municipal se debe a la dificultad que entraña la obtención del PIB en áreas subnacionales.

\section{b) Carga fiscal}

Es un índice que mide la relación entre la imposición y la renta libre de gastos necesarios. En el numerador aparecen los ingresos coactivos y en el denominador la renta menos el consuno necesario.

Es un índice más expresivo que el de presión tributaria y adecuado para las comparaciones intermunicipales $y$, sobre todo, para las internacionales.

CUADRO 8

\section{RESUMEN DE LOS VALORES DE PRESION Y ESFUERZO FISCAL DEL MUNICIPIO DE SEVILLA}

\begin{tabular}{c|c|c|c|c|c|c}
\hline \multirow{2}{*}{ Años } & \multicolumn{2}{|c|}{ Valores de presión fiscal municipal nacional } & \multicolumn{3}{|c}{ Valores de presión fiscal municipal de Sevilla } \\
\cline { 2 - 7 } & $\begin{array}{c}\text { Presión total } \\
\text { tributaria }\end{array}$ & $\begin{array}{c}\text { Esfuerzo fiscal } \\
\text { T. nacional }\end{array}$ & $\begin{array}{c}\text { Presión fiscal } \\
\text { financiera }\end{array}$ & $\begin{array}{c}\text { Presión fiscal } \\
\text { rributaria }\end{array}$ & $\begin{array}{c}\text { Esfuerzo fiscal } \\
\text { T. nacional }\end{array}$ & $\begin{array}{c}\text { Presión fiscal } \\
\text { financiera }\end{array}$ \\
\hline & & & & & & \\
1971 & 1,19 & 1,65 & 2,55 & 1,19 & 1,72 & 3,12 \\
1972 & 1,10 & 1,31 & 2,50 & 1,23 & 1,42 & 4,03 \\
1973 & 1,19 & 1,18 & 2,62 & 1,24 & 1,35 & 2,87 \\
1974 & 1,65 & 1,33 & 2,49 & 1,12 & 1,60 & 5,49 \\
1975 & 1,29 & 0,89 & 2,52 & 1,23 & 1,06 & 2,59 \\
1976 & 1,36 & 0,79 & 2,84 & 1,36 & 0,95 & 3,70 \\
1977 & 1,93 & 0,89 & 2,80 & 1,36 & 1,05 & 2,68 \\
1978 & 1,31 & 0,50 & 2,46 & 1,25 & 0,58 & 3,04 \\
1979 & 1,35 & 0,44 & 2,87 & 1,36 & 0,50 & 2,70 \\
\hline
\end{tabular}

FUENTE: Elaboración propia. 


\section{c) Esfuerzo fiscal}

Implica una superación de los anteriores. Pertenece al campo de la microeconomía. En la formulación de Frank es la razón existente entre presión fiscal y PIB per cápita, multiplicada por 100. Bird utiliza la fórmula presión fiscal partida por renta personal disponible per cápita, multiplicando el resultado por 100 .

El cuadro 8 expresa los resultados a que se ha llegado, siguiendo el correspondiente proceso estadístico para establecer los índices aludidos.

\section{CONCLUSION}

Las ideas expuestas constituyen un intento de someter a análisis económico los resultados anuales de las haciendas locales. Con los que las cuentas anuales pasarian de ser una formalidad legal comprobatoria de la gestión presupuestaria a un instrumento eficaz para la adopción de políticas económicas, a partir de las conclusiones de dicho análisis.

Es una innovación, ya iniciada en algunas Entidades Locales, que viene obligada por el volumen de recursos que mueven estas haciendas y por la propia dinámica económica actual que requiere disponer de nuevos instrumentos. Se cumple así el postulado de Schumpeter de que en Economía nada permanece estático y que lo normal y necesario es la expansión, la transmisión dinámica, «que revoluciona incesantemente desde el interior la estructura económica, destruyendo continuamente sus elementos envejecidos y creando continuamente unos elementos nuevos» (17).

(17) PIERRE MASSE: El plan o el antiazar, p. 65. Editorial Labor, S. A. Barcelona. 1966. 
